\title{
A new upper bound on the total domination number of a graph
}

\author{
${ }^{1}$ Michael A. Henning* and ${ }^{2}$ Anders Yeo \\ ${ }^{1}$ School of Mathematical Sciences \\ University of KwaZulu-Natal \\ Pietermaritzburg, 3209 South Africa \\ ${ }^{2}$ Department of Computer Science \\ Royal Holloway, University of London, Egham \\ Surrey TW20 OEX, UK
}

Submitted: Sep 7, 2006; Accepted: Sep 3, 2007; Published: Sep 7, 2007

\begin{abstract}
A set $S$ of vertices in a graph $G$ is a total dominating set of $G$ if every vertex of $G$ is adjacent to some vertex in $S$. The minimum cardinality of a total dominating set of $G$ is the total domination number of $G$. Let $G$ be a connected graph of order $n$ with minimum degree at least two and with maximum degree at least three. We define a vertex as large if it has degree more than 2 and we let $\mathcal{L}$ be the set of all large vertices of $G$. Let $P$ be any component of $G-\mathcal{L}$; it is a path. If $|P| \equiv 0(\bmod 4)$ and either the two ends of $P$ are adjacent in $G$ to the same large vertex or the two ends of $P$ are adjacent to different, but adjacent, large vertices in $G$, we call $P$ a 0-path. If $|P| \geq 5$ and $|P| \equiv 1(\bmod 4)$ with the two ends of $P$ adjacent in $G$ to the same large vertex, we call $P$ a 1 -path. If $|P| \equiv 3(\bmod 4)$, we call $P$ a 3 -path. For $i \in\{0,1,3\}$, we denote the number of $i$-paths in $G$ by $p_{i}$. We show that the total domination number of $G$ is at most $\left(n+p_{0}+p_{1}+p_{3}\right) / 2$. This result generalizes a result shown in several manuscripts (see, for example, J. Graph Theory 46 (2004), 207-210) which states that if $G$ is a graph of order $n$ with minimum degree at least three, then the total domination of $G$ is at most $n / 2$. It also generalizes a result by Lam and Wei stating that if $G$ is a graph of order $n$ with minimum degree at least two and with no degree- 2 vertex adjacent to two other degree- 2 vertices, then the total domination of $G$ is at most $n / 2$.
\end{abstract}

Keywords: bounds, path components, total domination number AMS subject classification: 05C69

\footnotetext{
${ }^{*}$ Research supported in part by the South African National Research Foundation and the University of KwaZulu-Natal.
} 


\section{Introduction}

In this paper, we continue the study of total domination in graphs which was introduced by Cockayne, Dawes, and Hedetniemi [5]. A total dominating set, abbreviated TDS, of a graph $G$ is a set $S$ of vertices of $G$ such that every vertex is adjacent to a vertex in $S$. Every graph without isolated vertices has a TDS, since $S=V(G)$ is such a set. The total domination number of $G$, denoted by $\gamma_{t}(G)$, is the minimum cardinality of a TDS. A TDS of $G$ of cardinality $\gamma_{t}(G)$ is called a $\gamma_{t}(G)$-set. Total domination in graphs is now well studied in graph theory. The literature on this subject has been surveyed and detailed in the two books by Haynes, Hedetniemi, and Slater [7, 8].

For notation and graph theory terminology we in general follow [7]. Specifically, let $G=(V, E)$ be a graph with vertex set $V$ of order $n=|V|$ and edge set $E$ of size $m=|E|$, and let $v$ be a vertex in $V$. The open neighborhood of $v$ is the set $N(v)=\{u \in V \mid u v \in E\}$. For a set $S \subseteq V$, its open neighborhood is the set $N(S)=\cup_{v \in S} N(v)$. If $Y \subseteq V$, then the set $S$ is said to totally dominate the set $Y$ if $Y \subseteq N(S)$. For a set $S \subseteq V$, the subgraph induced by $S$ is denoted by $G[S]$. We denote the degree of $v$ in $G$ by $d_{G}(v)$, or simply by $d(v)$ if the graph $G$ is clear from context. The minimum degree (resp., maximum degree) among the vertices of $G$ is denoted by $\delta(G)$ (resp., $\Delta(G)$ ). We denote a path on $n$ vertices by $P_{n}$ and a cycle on $n$ vertices by $C_{n}$.

\section{Known bounds on the total domination number}

The decision problem to determine the total domination number of a graph is known to be NP-complete. Hence it is of interest to determine upper bounds on the total domination number of a graph. In particular, for a connected graph $G$ with minimum degree $\delta \geq 1$ and order $n$, the problem of finding upper bounds on $\gamma_{t}(G)$ in terms of $\delta$ and $n$ has been studied. The known upper bounds on $\gamma_{t}(G)$ in terms of $\delta$ and $n$ are summarized in Table 1.

\begin{tabular}{||lll||}
\hline \hline$\delta(G) \geq 1$ & $\Rightarrow \gamma_{t}(G) \leq \frac{2}{3} n$ & if $n \geq 3$ and $G$ is connected \\
$\delta(G) \geq 2$ & $\Rightarrow \gamma_{t}(G) \leq \frac{4}{7} n$ & if $G \notin\left\{C_{3}, C_{5}, C_{6}, C_{10}\right\}$ and $G$ is connected \\
$\delta(G) \geq 3$ & $\Rightarrow \gamma_{t}(G) \leq \frac{1}{2} n$ \\
$\delta(G) \geq 4 \Rightarrow \gamma_{t}(G) \leq \frac{3}{7} n$ \\
$\delta(G)$ large & $\Rightarrow \gamma_{t}(G) \leq\left(\frac{1+\ln \delta}{\delta}\right) n$ \\
\hline \hline
\end{tabular}

Table 1. Upper bounds on the total domination number of a graph $G$. 
The result in Table 1 when $\delta$ is large is found using probabilistic methods in graph theory. It can easily be deduced from results of Alon [1] that this upper bound for large $\delta$ is nearly optimal. But what happens when $\delta$ is small? The problem then becomes more difficult.

The result in Table 1 when $\delta \geq 1$ is due to Cockayne et al. [5] and the graphs achieving this upper bound are characterized by Brigham, Carrington, and Vitray [3].

The result in Table 1 when $\delta \geq 2$ can be found in [9]. A characterization of the connected graphs of large order with total domination number exactly four-sevenths their order is also given in [9].

Chvátal and McDiarmid [4] and Tuza [13] independently established that every hypergraph on $n$ vertices and $m$ edges where all edges have size at least three has a transversal $T$ such that $4|T| \leq m+n$. As a consequence of this result about transversals in hypergraphs, we have the result in Table 1 for the case when $\delta \geq 3$. We remark that Archdeacon et al. [2] recently found an elegant one page graph theoretic proof of this upper bound of $n / 2$ when $\delta \geq 3$. Two infinite families of connected cubic graphs with total domination number one-half their orders are constructed in [6]. Using transversals in hypergraphs, the connected graphs with minimum degree at least three and with total domination number exactly one-half their order are characterized in [10].

The result when $\delta \geq 3$ has recently been strengthened by Lam and Wei [11].

Theorem 1 (Lam, Wei [11]) If $G$ is a graph of order $n$ with $\delta(G) \geq 2$ such that every component of the subgraph of $G$ induced by its set of degree-2 vertices has size at most one, then $\gamma_{t}(G) \leq n / 2$.

The result in Table 1 when $\delta \geq 4$ is due to Thomasse and Yeo [12]. Their proof uses transversals in hypergraphs. Yeo [14] showed that for connected graphs $G$ with minimum degree at least four equality is only achieved in this bound if $G$ is the relative complement of the Heawood graph (or, equivalently, the incidence bipartite graph of the complement of the Fano plane).

\section{Main Result}

Our aim in this paper is to present a new upper bound on the total domination number of a graph with minimum degree two. For this purpose, we introduce some additional notation.

We call a component of a graph a path-component if it is isomorphic to a path. A path-component isomorphic to a path $P_{i}$ on $i$ vertices we call a $P_{i}$-component.

We define a vertex as small if it has degree 2 , and large if it has degree more than 2. Let $G$ be a connected graph with minimum degree at least two and maximum degree at least three. Let $\mathcal{S}$ be the set of all small vertices of $G$ and $\mathcal{L}$ the set of all large vertices of $G$. Consider the graph $G-\mathcal{L}=G[\mathcal{S}]$ induced by the small vertices. Let $P$ be any component of $G-\mathcal{L}$; it is a path. If $|P| \equiv 0(\bmod 4)$ and either the two ends of $P$ are adjacent in $G$ to the same large vertex or the two ends of $P$ are adjacent to different, 
but adjacent, large vertices in $G$, we call $P$ a 0 -path. If $|P| \geq 5$ and $|P| \equiv 1(\bmod 4)$ with the two ends of $P$ adjacent in $G$ to the same large vertex, we call $P$ a 1-path. If $|P| \equiv 3(\bmod 4)$, we call $P$ a 3 -path. For $i \in\{0,1,3\}$, we denote the number of $i$-paths in $G$ by $p_{i}(G)$, or simply by $p_{i}$ if the graph $G$ is clear from context. If $G^{\prime}$ is a graph, then for $i \in\{0,1,3\}$ we denote $p_{i}\left(G^{\prime}\right)$ simply by $p_{i}^{\prime}$. For notational convenience, for a graph $G$ of order $n$ and a graph $G^{\prime}$ of order $n^{\prime}$ we let

$$
\psi(G)=\frac{1}{2}\left(n+p_{0}+p_{1}+p_{3}\right) \quad \text { and } \quad \psi\left(G^{\prime}\right)=\frac{1}{2}\left(n^{\prime}+p_{0}^{\prime}+p_{1}^{\prime}+p_{3}^{\prime}\right) .
$$

We shall prove:

Theorem 2 If $G$ is a connected graph of order $n$ with $\delta(G) \geq 2$ and $\Delta(G) \geq 3$, then $\gamma_{t}(G) \leq \psi(G)$.

Note that Theorem 2 generalizes Theorem 1 (see [11]) and the result from Table 1 for $\delta(G) \geq 3($ see [4] and [13]).

\subsection{Preliminary Results and Observations}

Before presenting a proof of Theorem 2, we define three graphs which we call $X, Y$ and $Z$ shown in Figures $1(\mathrm{a})$, (b) and (c), respectively. The vertices named $x, y$ and $z$ in Figure 1 we call the link vertices of the graphs $X, Y$ and $Z$, respectively.

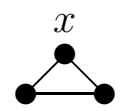

(a) $X$

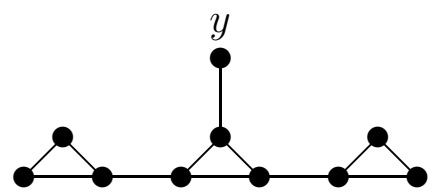

(b) $Y$

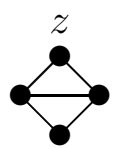

(c) $Z$

Figure 1: The three graphs $X, Y$ and $Z$.

Let $H \in\{X, Y, Z\}$. By attaching a copy of $H$ to a vertex $v$ in a graph $G$ we mean adding a copy of $H$ to the graph $G$ and joining $v$ with an edge to the link vertex of $H$. We call $v$ an attached vertex in the resulting graph. We will frequently use the following observations in the proof of Theorem 2 .

Observation 1 If $G^{\prime}$ is obtained from a graph $G$ with no isolated vertex by attaching a copy of $X$ with link vertex $x$ to a vertex $x^{\prime}$ of $G$, then there exists a $\gamma_{t}\left(G^{\prime}\right)$-set $S$ such that $S \cap\left(V(X) \cup\left\{x^{\prime}\right\}\right)=\left\{x, x^{\prime}\right\}$.

Observation 2 If $G^{\prime}$ is obtained from a graph $G$ with no isolated vertex by attaching a copy of $Y$ with link vertex $y$ to a vertex $y^{\prime}$ of $G$, then there exists a $\gamma_{t}\left(G^{\prime}\right)$-set $S$ that contains exactly four vertices of $Y$, namely the two vertices of $Y$ at distance 2 from $y$ and the two vertices of $Y$ at distance 3 from $y$ (and so, $y^{\prime}$ belongs to $S$ to totally dominate $y$ while a neighbor of $y^{\prime}$ in $G$ belongs to $S$ to totally dominate $\left.y^{\prime}\right)$. 
Observation 3 If $G^{\prime}$ is obtained from a graph $G$ with no isolated vertex by attaching a copy of $Z$ with link vertex $z$ to a vertex $z^{\prime}$ of $G$, then there exists a $\gamma_{t}\left(G^{\prime}\right)$-set $S$ that contains exactly two vertices of $Z$, namely $z$ and a neighbor of $z$ in $Z$ (and so, $z$ totally dominates $z^{\prime}$ in $\left.G^{\prime}\right)$.

We define an elementary 4-subdivision of a nonempty graph $G$ as a graph obtained from $G$ by subdividing some edge four times. We shall need the following lemma from [9].

Lemma 1 ([9]) Let $G$ be a nontrivial graph and let $G^{\prime}$ be obtained from $G$ by an elementary 4-subdivision. Then $\gamma_{t}\left(G^{\prime}\right)=\gamma_{t}(G)+2$.

We will refer to a graph $G$ as a reduced graph if $G$ has no induced path on six vertices, the internal vertices of which have degree 2 in $G$. Hence if $u, v_{1}, v_{2}, v_{3}, v_{4}, v$ is a path in a reduced graph $G$, then $d_{G}\left(v_{i}\right) \geq 3$ for at least one $i, 1 \leq i \leq 4$, or $u v \in E(G)$.

\subsection{Proof of Theorem 2}

We proceed by induction on the lexicographic sequence $\left(p_{0}+p_{1}+p_{3}, n\right)$, where $p_{0}+p_{1}+p_{3} \geq$ 0 and $n \geq 4$. For notational convenience, for a graph $G$ of order $n$ and a graph $G^{\prime}$ of order $n^{\prime}$, we denote the sequence $\left(p_{0}+p_{1}+p_{3}, n\right)$ by $s(G)$ and the sequence $\left(p_{0}^{\prime}+p_{1}^{\prime}+p_{3}^{\prime}, n^{\prime}\right)$ by $s\left(G^{\prime}\right)$. Further, we denote the set of small vertices of $G$ and $G^{\prime}$ by $\mathcal{S}$ and $\mathcal{S}^{\prime}$, respectively, and the set of large vertices of $G$ and $G^{\prime}$ by $\mathcal{L}$ and $\mathcal{L}^{\prime}$, respectively.

By Lemma 1, we may assume that $G$ is a reduced graph. Thus since $G$ is a connected graph with $\Delta(G) \geq 3$, every component of $G[\mathcal{S}]$ is a path $P_{i}$ for some $i$ where $1 \leq i \leq 5$.

When $p_{0}+p_{1}+p_{3}=0$, every component of $G[\mathcal{S}]$ is either $P_{1}$ or $P_{2}$ and the desired result follows from Theorem 1. This establishes the base case. Assume, then, that $p_{0}+p_{1}+p_{3} \geq 1$ and $n \geq 4$ and that for all connected graphs $G^{\prime}$ of order $n^{\prime}$ with $\delta\left(G^{\prime}\right) \geq 2$ and $\Delta\left(G^{\prime}\right) \geq 3$ that have lexicographic sequence $s\left(G^{\prime}\right)$ smaller than $s, \gamma_{t}\left(G^{\prime}\right) \leq \psi\left(G^{\prime}\right)$. Let $G=(V, E)$ be a connected graph of order $n$ with $\delta(G) \geq 2$ and $\Delta(G) \geq 3$ and with lexicographic sequence $s(G)=s$.

Observation 4 We may assume that $p_{0}=0$.

Proof. Suppose that $p_{0} \geq 1$. Let $P: v_{1}, v_{2}, v_{3}, v_{4}$ be a $P_{4}$-component of $G[\mathcal{S}]$. Let $u$ be the neighbor of $v_{1}$ not on $P$ and let $v$ be the neighbor of $v_{4}$ not on $P$.

Suppose firstly that $u \neq v$. Since $G$ is a reduced graph, $u v \in E(G)$. Let $G^{\prime}=G-V(P)$. Then, $G^{\prime}$ is a connected graph of order $n^{\prime}$ with $\delta\left(G^{\prime}\right) \geq 2$. Suppose $G^{\prime}$ is a cycle. Then, $G^{\prime} \in\left\{C_{3}, C_{4}, C_{5}, C_{6}\right\}$. If $G^{\prime}=C_{3}$, then $\gamma_{t}(G)=4$ and $\psi(G)=4$. If $G^{\prime}=C_{4}$, then $\gamma_{t}(G)=4$ and $\psi(G)=4 \frac{1}{2}$. If $G^{\prime}=C_{5}$, then $\gamma_{t}(G)=5$ and $\psi(G)=5 \frac{1}{2}$. If $G^{\prime}=C_{6}$, then $\gamma_{t}(G)=6$ and $\psi(G)=6$. In all cases, $\gamma_{t}(G) \leq \psi(G)$. Hence we may assume that $\Delta\left(G^{\prime}\right) \geq 3$. We remark that it is possible that the graph $G^{\prime}$ has an induced path on six vertices containing $u$ and $v$ with the internal vertices on this path having degree 2 in $G^{\prime}$, in which case $G^{\prime}$ is not a reduced graph, but then it is not a problem to reduce it. Since $p_{0}^{\prime}+p_{1}^{\prime}+p_{3}^{\prime} \leq p_{0}+p_{1}+p_{3}$ and $n^{\prime}=n-4$, the lexicographic sequence $s\left(G^{\prime}\right)$ is smaller 
than $s(G)$. Applying the inductive hypothesis to $G^{\prime}, \gamma_{t}\left(G^{\prime}\right) \leq \psi\left(G^{\prime}\right) \leq \psi(G)-2$. Every $\gamma_{t}\left(G^{\prime}\right)$-set can be extended to a TDS of $G$ by adding to it the vertices $\left\{v_{2}, v_{3}\right\}$, and so $\gamma_{t}(G) \leq \gamma_{t}\left(G^{\prime}\right)+2 \leq \psi(G)$.

Suppose secondly that $u=v$. Then, $C: v, v_{1}, v_{2}, v_{3}, v_{4}, v$ is a cycle in $G$. Let $G^{\prime}$ be the graph obtained from $G-V(C)$ by attaching the same copy of $Z$ to each vertex in $N_{G}(v) \backslash\left\{v_{1}, v_{4}\right\}$. Then, $G^{\prime}$ is a connected (reduced) graph of order $n^{\prime}=n-1$ with $\delta\left(G^{\prime}\right) \geq 2$ and $\Delta\left(G^{\prime}\right) \geq 3$ (as $v$ was a large vertex, $z$ is attached to at least one vertex and $\Delta(Z)=3)$. The components of $G^{\prime}\left[\mathcal{S}^{\prime}\right]$, other than the $P_{1}$-component consisting of the degree-2 vertex in the copy of $Z$, are precisely the components of $G[\mathcal{S}]$ minus the path-component $P$. Hence, $p_{0}^{\prime}=p_{0}-1, p_{1}^{\prime}=p_{1}$ and $p_{3}^{\prime}=p_{3}$. The lexicographic sequence $s\left(G^{\prime}\right)$ is therefore smaller than $s(G)$. Applying the inductive hypothesis to $G^{\prime}$, $\gamma_{t}\left(G^{\prime}\right) \leq \psi\left(G^{\prime}\right)=\psi(G)-1$. By Observation 3, there exists a $\gamma_{t}\left(G^{\prime}\right)$-set $S$ that contains the link vertex and a neighbor of the link vertex (distinct from the attached vertex) from the attached copy of $Z$. Deleting these two vertices in the attached copy of $Z$ from the set $S$ and adding to the resulting set the three vertices $v, v_{1}, v_{2}$ produces a TDS of $G$. Hence, $\gamma_{t}(G) \leq|S|+1=\gamma_{t}\left(G^{\prime}\right)+1 \leq \psi(G)$.

Observation 5 We may assume that $p_{1}=0$.

Proof. Suppose that $p_{1} \geq 1$. Let $P: v_{1}, v_{2}, \ldots, v_{5}$ be a $P_{5}$-component of $G[\mathcal{S}]$. Since $G$ is a reduced graph, $v_{1}$ and $v_{5}$ have a common neighbor $v$ in $G$. Let $G^{\prime}$ be obtained from $G$ by deleting the vertices $v_{3}, v_{4}$ and $v_{5}$ and adding the edge $v v_{2}$; that is, $G^{\prime}=$ $\left(G-\left\{v_{3}, v_{4}, v_{5}\right\}\right) \cup\left\{v v_{2}\right\}$. Then, $G^{\prime}$ is a reduced connected graph of order $n^{\prime}$ with $\delta\left(G^{\prime}\right) \geq 2$ and $\Delta\left(G^{\prime}\right)=\Delta(G) \geq 3$. Further, $p_{0}^{\prime}=p_{0}, p_{1}^{\prime}=p_{1}-1, p_{3}^{\prime}=p_{3}$, and $n^{\prime}=n-3$. Hence the lexicographic sequence $s\left(G^{\prime}\right)$ is smaller than $s(G)$. Applying the inductive hypothesis to $G^{\prime}, \gamma_{t}\left(G^{\prime}\right) \leq \psi\left(G^{\prime}\right)=\psi(G)-2$. Let $S^{\prime}$ be a $\gamma_{t}\left(G^{\prime}\right)$-set that contains neither $v_{1}$ nor $v_{2}$ (if there is a $\gamma_{t}\left(G^{\prime}\right)$-set $S^{\prime}$ that contains both $v_{1}$ and $v_{2}$, simply replace these two vertices in $S^{\prime}$ by $v$ and a neighbor of $v$ in $G-V(P)$, while if there is a $\gamma_{t}\left(G^{\prime}\right)$-set $S^{\prime}$ that contains exactly one of $v_{1}$ and $v_{2}$, simply replace this vertex in $S^{\prime}$ by a neighbor of $v$ in $G-V(P)$ ). Then, $S^{\prime} \cup\left\{v_{3}, v_{4}\right\}$ is a TDS of $G$, and so $\gamma_{t}(G) \leq\left|S^{\prime}\right|+2=\gamma_{t}\left(G^{\prime}\right)+2 \leq \psi(G)$.

By Observations 4 and 5 , we have $p_{0}=p_{1}=0$ and $p_{3} \geq 1$. Thus, since $G$ is a reduced graph, every component of $G[\mathcal{S}]$ is a path $P_{i}$ for some $i$ where $1 \leq i \leq 3$. Let $P: v_{1}, v_{2}, v_{3}$ be a $P_{3}$-component of $G[\mathcal{S}]$. Let $u$ be the neighbor of $v_{1}$ not on $P$ and let $v$ be the neighbor of $v_{3}$ not on $P$.

Observation 6 We may assume that $u \neq v$.

Proof. Suppose that $u=v$. Let $G^{\prime}$ be the graph obtained from $G-V(P)$ by attaching both a copy of $X$ and a copy of $Z$ to the vertex $v$. Then, $G^{\prime}$ is a connected (reduced) graph of order $n^{\prime}=n+4$ with $\delta\left(G^{\prime}\right) \geq 2$ and $\Delta\left(G^{\prime}\right)=\Delta(G) \geq 3$. The degree of the large vertex $v$ is unchanged in $G$ and $G^{\prime}$. Since $p_{0}^{\prime}=p_{0}=0, p_{1}^{\prime}=p_{1}=0$ and $p_{3}^{\prime}=p_{3}-1$, the lexicographic sequence $s\left(G^{\prime}\right)$ is smaller than $s(G)$. Applying the inductive hypothesis to $G^{\prime}, \gamma_{t}\left(G^{\prime}\right) \leq \psi\left(G^{\prime}\right)=\psi(G)+3 / 2$. By Observations 1 and 3, there exists a $\gamma_{t}\left(G^{\prime}\right)$-set $S$ that contains the vertex $v$ and three vertices from the attached copies of $X$ and $Z$, namely 
the link vertex and a neighbor of the link vertex in the attached copy of $Z$ and the link vertex in the attached copy of $X$. Deleting these three vertices in the attached copies of $X$ and $Z$ from the set $S$ and adding to the resulting set the vertex $v_{1}$ produces a TDS of $G$. Hence, $\gamma_{t}(G) \leq|S|-2=\gamma_{t}\left(G^{\prime}\right)-2 \leq \psi(G)-1 / 2$.

Observation 7 We may assume that no common neighbor of $u$ and $v$ has degree two.

Proof. Suppose that $u$ and $v$ have a common neighbor $w$ with $N(w)=\{u, v\}$. Let $W$ be the set of all such degree- 2 vertices that are adjacent to both $u$ and $v$. Let $R=$ $W \cup\left\{u, v, v_{1}, v_{2}, v_{3}\right\}$. Let $N_{u v}=(N(u) \cup N(v)) \backslash R$.

Suppose $V=R$. If $|W|=1$, then $u v \in E, n=6, p_{3}=1$, and $\gamma_{t}(G)=3=\psi(G)-1 / 2$. If $|W| \geq 2$, then $n \geq 7, p_{3}=1$, and $\gamma_{t}(G) \leq 4 \leq \psi(G)$. Hence we may assume that $V \neq R$. Thus, $\left|N_{u v}\right| \geq 1$. At least one of $u$ and $v$, say $v$, is therefore adjacent to a vertex in $V \backslash R$.

If $|W| \geq 2$, then let $G^{\prime}=G-w$. The graph $G^{\prime}$ is a connected reduced graph of order $n^{\prime}=n-1$ with $\delta\left(G^{\prime}\right) \geq 2$ and $\Delta\left(G^{\prime}\right) \geq d_{G}(v)-1 \geq 3$. If $d_{G^{\prime}}(u)=2$, then $p_{0}^{\prime}=p_{0}, p_{1}^{\prime}=p_{1}+1$ and $p_{3}^{\prime}=p_{3}-1$, while if $d_{G^{\prime}}(u) \geq 3$, then $p_{0}^{\prime}=p_{0}, p_{1}^{\prime}=p_{1}$ and $p_{3}^{\prime}=p_{3}$. In both cases, $p_{0}^{\prime}+p_{1}^{\prime}+p_{3}^{\prime}=p_{0}+p_{1}+p_{3}$. Applying the inductive hypothesis to $G^{\prime}, \gamma_{t}\left(G^{\prime}\right) \leq \psi\left(G^{\prime}\right)=\psi(G)-1 / 2$. Every $\gamma_{t}\left(G^{\prime}\right)$-set is a TDS of $G$, and so $\gamma_{t}(G) \leq \gamma_{t}\left(G^{\prime}\right)<\psi(G)$. Hence we may assume that $|W|=1$, and so $W=\{w\}$ and $R=\left\{u, v, v_{1}, v_{2}, v_{3}, w\right\}$.

Let $G^{\prime}$ be the connected graph obtained from $G-R$ by attaching the same subgraph $X$ to every vertex in $N_{u v}$. Let $N_{u v}^{*}=(N(u) \cap N(v)) \backslash R$ and if $N_{u v}^{*} \neq \emptyset$ then also attach the same subgraph $Z$ to every vertex in $N_{u v}^{*}$. Note that $d_{G^{\prime}}(x)=d_{G}(x)$ for every vertex $x \in V\left(G^{\prime}\right) \backslash V(X \cup Z)$. Furthermore, $\Delta\left(G^{\prime}\right) \geq 3$ as the link vertex in the copy of $X$ has degree at least three. The components of $G^{\prime}\left[\mathcal{S}^{\prime}\right]$, other than the $P_{2}$-component consisting of the two degree- 2 vertices in the copy of $X$ and, if $N_{u v}^{*} \neq \emptyset$, the $P_{1}$-component consisting of the degree- 2 vertex in the copy of $Z$, are precisely the components of $G[\mathcal{S}]$ minus the path-component $P$ and the $P_{1}$-component consisting of the vertex $w$. Hence, $p_{0}^{\prime}=p_{0}=0$, $p_{1}^{\prime}=p_{1}=0$ and $p_{3}^{\prime}=p_{3}-1$. Thus, $p_{0}^{\prime}+p_{1}^{\prime}+p_{3}^{\prime}=p_{0}+p_{1}+p_{3}-1$. Applying the inductive hypothesis to $G^{\prime}, \gamma_{t}\left(G^{\prime}\right) \leq \psi\left(G^{\prime}\right)$. By the construction of $X$, there exists a $\gamma_{t}\left(G^{\prime}\right)$-set $S$, such that $S \cap N_{u v} \neq \emptyset$ and $|S \cap X|=1$. We may assume without loss of generality that $v$ is adjacent in $G$ to a vertex in $S \cap N_{u v}$.

On the one hand, suppose that $N_{u v}^{*} \neq \emptyset$. Then, $n^{\prime}=n+1$ and $\psi\left(G^{\prime}\right)=\psi(G)$. Delete from $S$ the vertices in $X$ and $Z$ and add the vertices $\left\{u, v, v_{1}\right\}$. The resulting set has size at most that of $S$ and is a TDS of $G$. Hence, $\gamma_{t}(G) \leq \gamma_{t}\left(G^{\prime}\right) \leq \psi\left(G^{\prime}\right)=\psi(G)$.

On the other hand, suppose that $N_{u v}^{*}=\emptyset$. Then, $n^{\prime}=n-3$ and $\psi\left(G^{\prime}\right)=\psi(G)-2$. Now delete from $S$ the vertex in $X$ and add the vertices $\left\{u, v, v_{1}\right\}$. The resulting set has size $|S|+2$ and is a TDS of $G$. Hence, $\gamma_{t}(G) \leq \gamma_{t}\left(G^{\prime}\right)+2 \leq \psi\left(G^{\prime}\right)+2=\psi(G)$.

Let $R=\left\{u, v, v_{1}, v_{2}, v_{3}\right\}$ and let $N_{u v}=(N(u) \cup N(v)) \backslash R$. Then, $\left|N_{u v}\right| \geq 1$. By Observation 7 , every vertex in $N_{u v}$ that is adjacent to both $u$ and $v$ has degree at least 3 . Hence every vertex in $N_{u v}$ is adjacent to at least one vertex different from $u$ and $v$. 
Observation 8 We may assume that $\left|N_{u v}\right|=1$.

Proof. Suppose that $\left|N_{u v}\right| \geq 2$. Let $G^{\prime}$ be obtained from $G-V(P)$ by adding all possible edges between the set $\{u, v\}$ and the set $N_{u v}$, and by adding the edge $u v$ if $u$ and $v$ are not adjacent to $G$. Then, $G^{\prime}$ is a connected (reduced) graph of order $n^{\prime}=n-3$ with $\delta\left(G^{\prime}\right) \geq 2$ and $\Delta\left(G^{\prime}\right) \geq 3$. By construction, both $u$ and $v$ are large vertices in $G^{\prime}$. Note that some vertices in $N_{u v}$ may be large in $G^{\prime}$ even though they were not large in $G$. However as every component in $G[\mathcal{S}]$ is a path containing at most three vertices, we note that $p_{0}^{\prime}+p_{1}^{\prime}+p_{3}^{\prime} \leq p_{0}+p_{1}+p_{3}-1$. We can therefore apply the inductive hypothesis to $G^{\prime}$. Thus, $\gamma_{t}\left(G^{\prime}\right) \leq \psi\left(G^{\prime}\right) \leq \psi(G)-2$. Let $S^{\prime}$ be a $\gamma_{t}\left(G^{\prime}\right)$-set. If $\{u, v\} \subseteq S^{\prime}$, let $S=S^{\prime} \cup\left\{v_{1}, v_{3}\right\}$. If $\left|\{u, v\} \cap S^{\prime}\right| \leq 1$, then the set $S^{\prime}$ contains a vertex $u^{\prime} \in N_{u v}$ to totally dominate $u$ or $v$ in $G^{\prime}$. The vertex $u^{\prime}$ is adjacent in $G$ to at least one of $u$ and $v$, say to $u$. If $\left|\{u, v\} \cap S^{\prime}\right|=1$, let $S=S^{\prime} \cup\left\{u, v, v_{3}\right\}$. If $\{u, v\} \cap S^{\prime}=\emptyset$, let $S=S^{\prime} \cup\left\{v_{2}, v_{3}\right\}$. In all three cases, $S$ is a TDS of $G$ and $|S|=\left|S^{\prime}\right|+2$. Hence, $\gamma_{t}(G) \leq|S|=\gamma_{t}\left(G^{\prime}\right)+2 \leq \psi(G)$.

By Observation 8, $\left|N_{u v}\right|=1$, implying that $u v \in E$. Let $N_{u v}=\{w\}$. Let $G^{\prime}=$ $G-V(P)$. Then, $G^{\prime}$ is a connected (reduced) graph of order $n^{\prime}=n-3$ with $\delta\left(G^{\prime}\right) \geq 2$ and $\Delta\left(G^{\prime}\right)=\Delta(G) \geq 3$. Since $p_{0}^{\prime}+p_{1}^{\prime}+p_{3}^{\prime}=p_{0}+p_{1}+p_{3}-1$, we can apply the inductive hypothesis to $G^{\prime}$. Thus, $\gamma_{t}\left(G^{\prime}\right) \leq \psi\left(G^{\prime}\right)=\psi(G)-2$. Let $S^{\prime}$ be a $\gamma_{t}\left(G^{\prime}\right)$. Then, $S^{\prime} \cup\left\{v_{1}, v_{2}\right\}$ is a TDS of $G$, and so $\gamma_{t}(G) \leq\left|S^{\prime}\right|+2=\gamma_{t}\left(G^{\prime}\right)+2=\psi(G)$.

\subsection{Sharpness of Theorem 2}

To illustrate that the bound in Theorem 2 is sharp, we introduce a family $\mathcal{G}$ of graphs. For this purpose, we define three types of graphs which we call units.

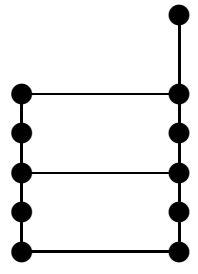

(i) Type-0

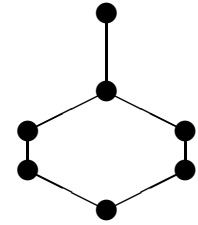

(ii) Type-1

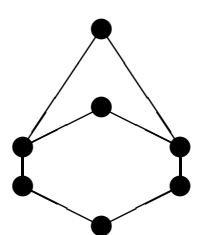

(iii) Type-3

Figure 2: The three types of units

We define a type-0 unit to be the graph obtained from a 10-cycle by adding a chord joining two vertices at maximum distance 5 apart on the cycle and then adding a pendant edge to a resulting vertex that has no degree-3 neighbor. We define a type-1 unit to be the graph obtained from a 6 -cycle by adding to this cycle a pendant edge. We define a type-3 unit to be the graph obtained from a 6-cycle by adding to this cycle a new vertex and joining it to two vertices at distance 2 on this cycle. The three types of units are shown in Figure 2. 
Next we define a link vertex in each unit as follows. In a type-0 unit and type- 1 unit, we call the degree- 1 vertex in the unit the link vertex of the unit, while in a type- 3 unit we select one of the two degree- 2 vertices with both its neighbors of degree 3 and call it the link vertex of the unit.

Let $\mathcal{G}$ denote the family of all graphs $G$ that are obtained from the disjoint union of at least two units, each of which is of type-0, type- 1 or type-3, in such a way that $G$ is connected and every added edge joins two link vertices. A graph $G$ in the family $\mathcal{G}$ is illustrated in Figure 3 (here the subgraph of $G$ induced by the link vertices is a cycle $C_{4}$ ).

The graph $G$ in Figure 3 has order $n=32, p_{0}=1, p_{1}=1, p_{3}=2$, and $\gamma_{t}(G)=18=$ $\psi(G)$. In general, if $G \in \mathcal{G}$ and $i \in\{0,1,3\}$, then each type- $i$ unit in $G$ contains an $i$-path and contributes one to $p_{i}$. Thus if $G \in \mathcal{G}$ has $a$ type- 0 units, $b$ type- 1 units, and $c$ type-3 units, then $n=11 a+7(b+c), p_{0}=a, p_{1}=b, p_{3}=c$ and $\gamma_{t}(G)=6 a+4(b+c)=\psi(G)$.

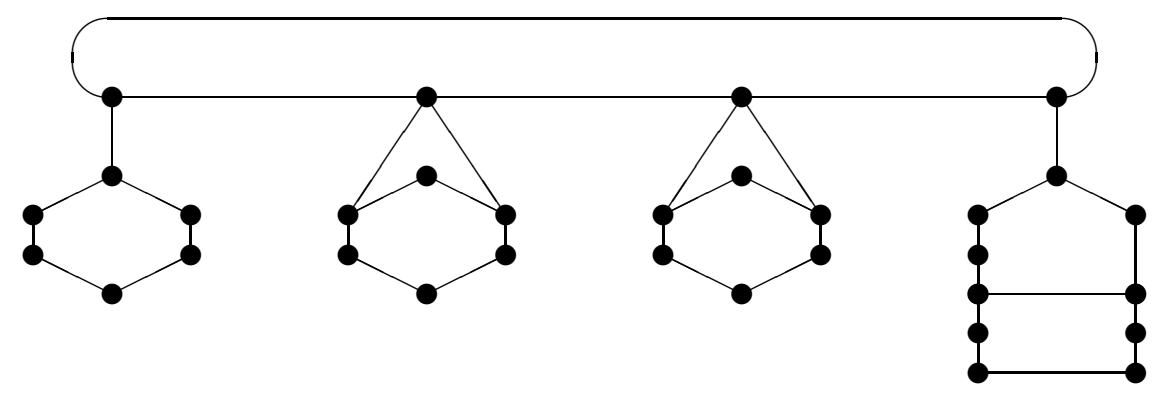

Figure 3: A graph $G$ in the family $\mathcal{G}$.

\section{References}

[1] N. Alon, Transversal number of uniform hypergraphs. Graphs Combin. 6 (1990), 1-4.

[2] D. Archdeacon, J. Ellis-Monaghan, D. Fischer, D. Froncek, P.C.B. Lam, S. Seager, B. Wei, and R. Yuster, Some remarks on domination. J. Graph Theory 46 (2004), 207-210.

[3] R.C. Brigham, J.R. Carrington, and R.P. Vitray, Connected graphs with maximum total domination number. J. Combin. Comput. Combin. Math. 34 (2000), 81-96.

[4] V. Chvátal and C. McDiarmid, Small transversals in hypergraphs. Combinatorica 12 (1992), 19-26.

[5] E. J. Cockayne, R. M. Dawes, and S. T. Hedetniemi, Total domination in graphs. Networks 10 (1980), 211-219.

[6] O. Favaron, M.A. Henning, C.M. Mynhardt, and J. Puech, Total domination in graphs with minimum degree three. J. Graph Theory 34 (2000), 9-19.

[7] T. W. Haynes, S. T. Hedetniemi, and P. J. Slater (eds), Fundamentals of Domination in Graphs, Marcel Dekker, Inc. New York, 1998.

[8] T. W. Haynes, S. T. Hedetniemi, and P. J. Slater (eds), Domination in Graphs: Advanced Topics, Marcel Dekker, Inc. New York, 1998. 
[9] M. A. Henning, Graphs with large total domination number. J. Graph Theory 35 (2000), 21-45.

[10] M. A. Henning and A. Yeo, Hypergraphs with large transversal number and with edge sizes at least three, manuscript (2006).

[11] P. C. B. Lam and B. Wei, On the total domination number of graphs. Utilitas Math. 72 (2007), 223-240.

[12] S. Thomassé and A. Yeo, Total domination of graphs and small transversals of hypergraphs. To appear in Combinatorica.

[13] Z. Tuza, Covering all cliques of a graph. Discrete Math. 86 (1990), 117-126.

[14] A. Yeo, Improved bound on the total domination in graphs with minimum degree four, manuscript (2006). 